\title{
Characterisation of additively manufactured metal surfaces by means of X-ray computed tomography and generalised surface texture parameters
}

\author{
a Department of Management and Engineering, University of Padua, Vicenza, Italy \\ ${ }^{b}$ EPSRC Future Metrology Hub, University of Huddersfield, Huddersfield, UK \\ ${ }^{c}$ Department of Industrial Engineering, University of Padua, Padua, Italy
}

Filippo Zanini a, Luca Pagani b, Enrico Savio (1)c, Simone Carmignato (2)a

X-ray computed tomography (CT) has recently started to be used for evaluating the surface topography of metal parts produced by additive manufacturing (AM). In particular, CT can overcome the main limitations of contact and optical measuring techniques, as CT enables non-destructive measurements of both internal and difficult-to-access surfaces, including micro-scale re-entrant surface features. This work aims at improving the understanding of CT-based surface topography characterisation, including the use of new generalised surface texture parameters suited for AM surfaces. Experimental investigations are performed on Ti6Al4V reference samples fabricated by powder bed fusion to determine the uncertainty of CT surface topography measurements.

Metrology; Additive manufacturing; X-ray computed tomography

\section{Introduction}

Additive manufacturing (AM) of metals enables the production of highly customised industrial parts characterised by complex external and/or internal geometries (e.g. freeform surfaces) that cannot be manufactured using conventional processes [1]. However, such parts typically exhibit surface topographies with complex and intricate re-entrant features as well as high roughness, which can (i) limit the adoption of AM processes for precision engineering components and (ii) lead to significant deviations when performing dimensional measurements using different measuring techniques [2].

Optical areal measuring techniques - such as coherence scanning interferometry, focus variation and confocal microscopy - are currently used to evaluate the topography of AM complex surfaces [3]. However, significant deviations were observed when comparing the areal surface texture parameters (defined in ISO 25178-2 [4]) and the surface profiles obtained from different optical instruments [5]. Unlike the surfaces produced via conventional manufacturing techniques, AM surfaces are characterised by a significant presence of micro-scale re-entrant features, which are in most cases difficult or impossible to be accessed using optical or contact techniques. In addition, optical and contact instruments cannot be used for non-destructive inspection of internal surfaces, such as the surfaces of internal channels whose fabrication is enabled by AM processes [6].

Industrial X-ray computed tomography (CT), which is already successfully used for dimensional quality control of AM parts [7], has recently started to be considered also as a viable technique for AM surface topography evaluation [8]. In particular, currently available CT systems are capable of overcoming the main limitations of contact and optical measuring techniques, as they can be used to measure external as well as internal and difficultto-accesses AM surfaces, non-destructively and with adequate metrological structural resolution [9]. CT-based measurements of AM surface topographies have already been compared with optical areal measurements [5] in terms of both areal [4] and profile [10] surface texture parameters, which can be computed on CT data after removal of all re-entrant surface features. In fact, errors occur if there is more than one surface point to be projected onto the reference plane at a same location [8]. However, such type of comparison is limited by the fact that a number of surface features actually acquired by CT are filtered out, hence discarding potentially relevant surface information. In order to overcome this limitation and to improve the topography characterisation of metal AM surfaces, this paper presents a methodology based on the computation of generalised surface texture parameters on CT data without removing re-entrant surface features. This enables a more consistent quantitative characterisation of such surfaces and the investigation of surface functionality when influence by re-entrant features can be relevant. Moreover, the accuracy of CT surface topography measurements, already investigated by Zanini et al. [6] in the case of conventional parameters computed on CT data without reentrant features, is here evaluated in the case of the methodology introduced in this paper using reference AM samples characterised by calibrated surface profiles representing the actual morphology, including re-entrant features.

\section{Definition of generalised surface texture parameters}

To compute the surface texture parameters on the actual CT data without removing the re-entrant features, a generalised formulation of texture parameters is used in this work, based on a previous work by Pagani et al. [11]. For the sake of simplicity, the formulas in the following are related to surface profiles, however the same concepts are applicable to surface topographies. The proposed generalisation is based on the parameterisation of the surface or profile containing re-entrant features. In the case of profiles, the parameterisation is conducted by computing the infinitesimal arc length elements of consecutive segments along the investigated profile. If no re-entrant features are present, then these parameters coincide with the corresponding conventional ones. In this work, the parameters (namely P-parameters, [10]) 
are evaluated on the primary profile, i.e. the total profile after the form profile is removed.

Let the measured profile be represented by a parametric curve $\boldsymbol{r}(t)$, it can be defined as:

$\boldsymbol{r}(t)=\boldsymbol{r}_{F}(t)+r_{\text {res }}(t) \cdot \boldsymbol{n}_{F}(t)$

where $\boldsymbol{r}_{F}(t)$ is the form profile (e.g. mean line, computed using a total least squares method) and $r_{r e s}(t) \cdot \boldsymbol{n}_{F}(t)$ represents the primary profile. $r_{\text {res }}(t)$ is the orthogonal signed distance between the measured and the mean line, while $\boldsymbol{n}_{F}(t)$ is the normal of the mean line.

Figure 1(a) shows a typical AM surface profile (obtained from experimental data used in this work; see Section 4) before and after removal of re-entrant features, and the respective mean lines computed using a total least squares method. It can be observed that the two obtained mean lines are significantly different.

The profile texture parameters are computed re-parameterising the mean line according to the arc length parameterisation. The height parameters can then be computed as the integral of a scalar field on the mean line. For example, the arithmetical mean deviation can be computed as:

$P a=\frac{1}{L_{F}} \int_{r_{F}(t)}\left|r_{\text {res }}(t)\right| d l$

where $d l=\left\|\boldsymbol{r}_{F}^{\prime}(t)\right\| d t$ is the infinitesimal arc length element and $L_{F}=\int_{r_{F}(t)} d l$ is the length of the projection of the measured profile on the mean line. The other height parameters $(P q, P v$, $P p, P t, P s k$ and $P k u$ ) can be similarly computed, while the root mean square slope (i.e. mean gradient $[4,10]$ ) can be obtained from the following formula:

$P d q=\sqrt{\frac{1}{L_{F}} \int_{r_{F}(t)}\left(\frac{r_{r e s}^{\prime}(t)}{\left\|\boldsymbol{r}_{F}^{\prime}(t)\right\|}\right)^{2} d l}$

Another set of parameters can be defined after computing the material ratio curve of the profile (Abbott Firestone curve [12], named 'AF curve' in the following). The AF curve is computed starting from the area $(A)$ below the profile, subdividing the contribution of each quadrature point along the height. The area related parameters are $P m p, P m c, P v c$ and $P v v$ (corresponding to the ones defined in ISO 25178-2 [4]). Figure 1(b) compares the AF curves obtained from the AM surface profile presented in Figure 1(a), before and after removing the re-entrant features.

For a quantitative evaluation of the complexity of an AM surface, a new parameter $(\operatorname{Pr} f)$ is introduced in this work to evaluate the percentage of re-entrant features. It is defined as:

$\operatorname{Prf}=\frac{L_{F}-L_{S}}{2 L_{S}}$

where $L_{S}$ is the length of the projection on the mean line of the measured profile after removing the re-entrant features.

\section{Comparison of generalised and conventional parameters}

The importance of including the re-entrant surface features on the computation of surface texture parameters is here proven by comparing the parameters calculated using the definition proposed in Section 2 (re-entrant features not removed) with the corresponding conventional parameters (re-entrant features removed). The AM surface profile represented in Figure 1(a) is used as an example for the comparison of P-parameters. Table 1 shows the obtained percentage differences. Concerning $P a, P q, P p$ and $P v$, the high differences are due to the fact that their computation is based on the position of the mean line, which changes consistently when removing the re-entrant features from the actual profile (see Figure 1). The percentage difference is high especially for $P p$ because the mean line is shifted towards the profile peaks after removing the re-entrant features. Regarding $P v$, the mean line is shifted further from the profile valleys but the information on some valleys is discarded when removing the reentrant features as seen in Figure 1, hence leading to a smaller relative error with respect to $P p$. Pt is reduced after removing the re-entrant features due to the missing information about the "shadowed" valleys. In the example, Psk is also strongly affected by the different description of the valleys, which can change drastically the symmetry of the profile. $P k u$ is connected to the profile steepness, which is slightly modified due to a different description of the profile peaks (i.e. outward directed portion of the assessed profile connecting two adjacent points of the intersection of the profile with the mean line). $P d q$ exhibits a high deviation due to the different smoothness of the profile: if the reentrant features are not removed, the profile's curvature is smoother. The area $A$ measured below the two profiles is larger when removing the undercuts, as can be noticed in Figure 1. Concerning the AF curves (Figure 1(b)), there is a substantial difference that leads to deviations between the related generalised and conventional parameters (i.e. Pmc and $P v c$ in Table 1).

Figure 2(a) illustrates an AM surface topography acquired by Xray computed tomography, after form suppression (mean plane in this case). A very large and deep re-entrant surface feature that would not have been detected using optical or contact techniques - can be observed (Figure 2(b)). The generalised areal surface texture parameters (S-parameters) were compared to the corresponding conventional ones after removing the re-entrant features. High differences, in the order of those obtained for profiles, were obtained. Considerations already reported for profile surface texture parameters are valid in this case as well.
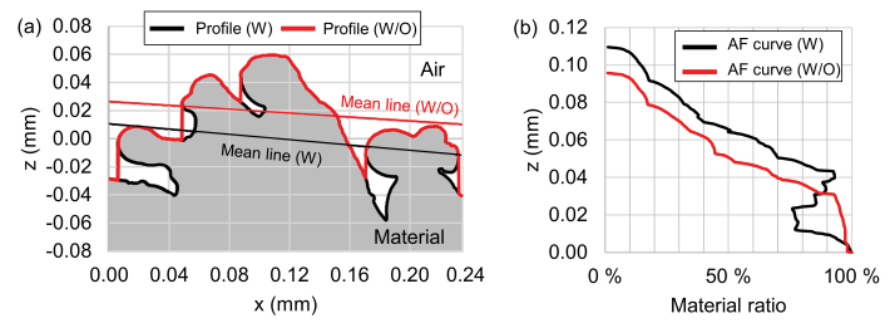

Figure 1. (a) Portion of an actual AM surface profile (black profile, "W" stands for "with re-entrant features") and the same profile after removing the re-entrant features (red profile, "W/O" stands for "without re-entrant features"); the mean line is depicted for each profile. (b) AF curves obtained from the two profiles.

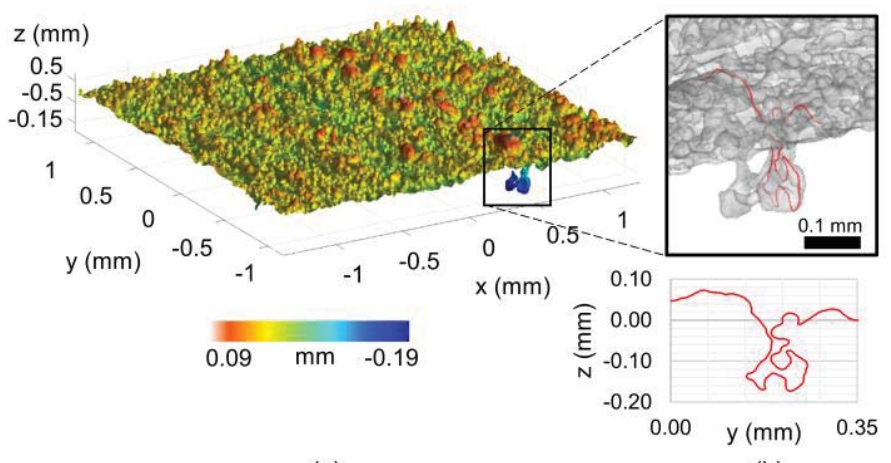

(a)

(b)

Figure 2. (a) AM surface topography extracted from CT data; (b) zoom on the deepest re-entrant feature.

Table 1. Values of the P-parameters computed according to the proposed method (on profiles having re-entrant features) and percentage differences between generalised P-parameters and corresponding conventional parameters (computed on the same profiles after removing all the re-entrant features). All the values are expressed in micrometres, except for $P s k$ and $P k u$ (dimensionless) and $A$ that is expressed in squared micrometres.

\begin{tabular}{|c|c|c|c|c|c|c|c|c|c|c|c|}
\hline P-parameter & $P a$ & $P q$ & $P p$ & $P v$ & $P t$ & Psk & $P k u$ & $P d q$ & $P m c$ & $P v c$ & $A$ \\
\hline Value & 21.6 & 26.4 & 62.4 & 47.2 & 109.6 & 0.34 & 2.45 & 2.2 & 39.4 & 48.3 & 0.3 \\
\hline Difference (\%) & 20 & 19 & 31 & -8 & 13 & 85 & -14 & -212 & 13 & 12 & 13 \\
\hline
\end{tabular}




\section{Method to verify the accuracy of CT measurements}

For assessing the accuracy of AM surface topography measurements performed by X-ray CT, the procedure proposed by Zanini et al. [6] and illustrated in Figure 3 was followed. Reference samples (parallelepipeds with nominal dimensions equal to $5 \times 5 \times 10 \mathrm{~mm}$ ) were fabricated by laser-powder bed fusion (L-PBF) of Ti6Al4V, using a Sisma MYSINT100 AM system. Markers (i.e. cylindrical holes and planar slots) were micro-milled on the surfaces of interest to enable the accurate alignment of different CT volumes. In particular, the samples were CT scanned using a metrological CT system (Nikon Metrology MCT225), characterised by a micro-focus X-ray source (minimum focal spot size: $3 \mu \mathrm{m})$, 16-bit detector with $2000 \times 2000$ pixels, highprecision linear guideways, temperature controlled cabinet $(20 \pm$ $0.5{ }^{\circ} \mathrm{C}$ ), and maximum permissible error (MPE) for length measurements equal to $9 \pm(L / 50) \mu \mathrm{m}$ (with measured length $L$ expressed in $\mathrm{mm}$ ). The following parameters were used to conduct three repeated scans: tube voltage $190 \mathrm{kV}$, current $36 \mu \mathrm{A}$, exposure time $2 \mathrm{~s}, 1800$ projections, and five different voxel sizes: $4,6,8,10$ and $12 \mu \mathrm{m}$. A beam hardening software correction $\left(y=1.32 \cdot\left(0.25 x^{2}+0.75 x\right)\right)$ and a median filter $(3 \times 3$ kernel size $)$ were applied during the reconstruction of the CT-based model.

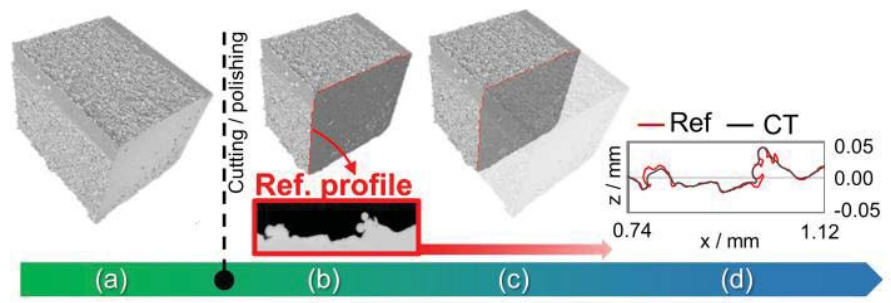

Figure 3. Experimental procedure: (a) CT scan of Ti6Al4V L-PBF samples before cutting, (b) CT scan after cutting and acquisition of reference profiles using an imaging probing system, (c) alignment of CT volumes pre- and post-cut, (d) comparison between CT profiles and reference profiles.

A face-milling operation followed by epoxy-based embedding and fine polishing were performed to (i) cut the samples in correspondence to a plane perpendicular to the analysed surfaces and (ii) acquire the coordinates of points of 2D reference crosssectional profiles to be calibrated, using a multisensor coordinate measuring machine (CMM) equipped with imaging probing sensor (Werth Video-Check IP 400). The MPE for length measurements is equal to $1.8 \pm(L / 250) \mu \mathrm{m}$ (with length $L$ expressed in $\mathrm{mm}$ ).

A new CT scan was then performed on the cut sample using the same parameters listed above, with the purpose to align the CT volumes acquired before and after the cutting/polishing procedure, to identify in the CT data the corresponding profiles calibrated by the imaging probing system. The uncertainty of CT surface topography measurements is then determined as described below in Section 5.2.

\section{Investigation on the accuracy of CT measurements}

\subsection{Effect of magnification}

The voxel size $(v s)$ is one of the factors having the most significant influence on the metrological structural resolution [9], upon which depends the CT capability of measuring micro-scale topographical details (including re-entrant features). Moreover, in conventional cone-beam CT scanning (as in the case of the CT system used in this work), the magnification factor $(m)$ is geometrically obtained by dividing the source-to-detector distance $(S D D)$ by the source-to-object distance $(S O D)$. The closer the object is to the source, the higher is the geometrical magnification, hence the smaller is the resulting voxel size, according to equation (5):

$v s=p s \cdot m=p s \cdot(S D D / S O D)$

where $p s$ is the detector pixel size. The dimensions of the imaged object part should be small enough to reach very small voxel sizes (hence higher metrological structural resolution) keeping, at the same time, the part within the X-ray detector field of view. Consequently, the maximum allowed dimension $(D \max )$ for the part to be scanned depends on the voxel size and on the number of pixels on a detector row $(n p)$ of the detector, as described by the following equation:

$D \max =v s \cdot n p=p s \cdot(S D D / S O D) \cdot n p$.

For the above-mentioned reasons, the effect of the voxel size is specifically addressed in this paper. In particular, the generalised profile parameters proposed in this work were computed on surface profiles measured by CT with 5 different voxel dimensions: 4, 6, 8, 10 and $12 \mu \mathrm{m}$ and then compared with reference values.

Figure 4 provides a visual comparison of one profile taken as an example, measured by (i) CT at $v s=4 \mu \mathrm{m}$, (ii) CT at $v s=12 \mu \mathrm{m}$, (iii) confocal microscope, and (iv) compared with the corresponding reference profile.

The diagram reported in Figure 5 plots the percentage deviations obtained for each investigated parameter between the measured profiles and reference profile (see Figure 4). An almost linear dependency can be observed between voxel size and the major part of investigated parameters. $P d q$ exhibited errors (increasing for bigger voxel sizes) up to $80 \%$ (negative); they are not displayed in Figure 5 for a better visualization. Results show that errors below $10 \%$ were obtained for the majority of the investigated parameters with voxel size equal or lower than $6 \mu \mathrm{m}$ (corresponding to a maximum sample size of approximately 12 $\mathrm{mm}$ for the CT system used in this work, that is equipped with an X-ray detector with $2000 \times 2000$ grid of pixels, with pixel size equal to $0.2 \mathrm{~mm}$ ). Moreover, the considered profile has a Prf equal to $6.2 \%$. With the scanning parameters used in this work, CT reached a maximum $\operatorname{Prf}$ of 2.2 . \% with the voxel size equal to 4 $\mu \mathrm{m}$ (further decreasing with larger voxel sizes). As it can be observed in Figure 4, the CT profile obtained with $v s=4 \mu \mathrm{m}$ approximates well with the reference profile but there is still a smoothing effect (due to the voxel dimension and to the median filter used for noise reduction) that limits the capability of

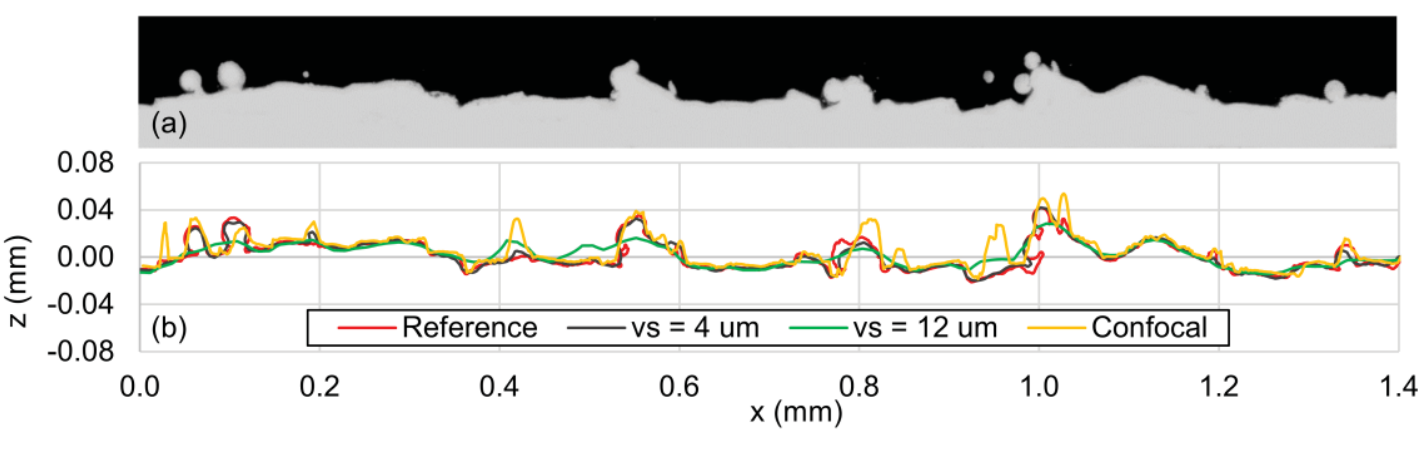

Figure 4. Portion of a cut section imaged using the imaging probing system described in Section 3.1 (a). Isolated particles are reentrant features coming out from the orthogonal direction. Cross-sectional profiles measured by CT at the highest and lowest magnification and by 4 confocal microscopy, compared to the reference profile (b). 
measuring very small re-entrant features. However, CT (at the highest resolution) provides a better description of the actual surface profile with respect to optical techniques (e.g. confocal microscopy, as seen in Figure 4(b)).

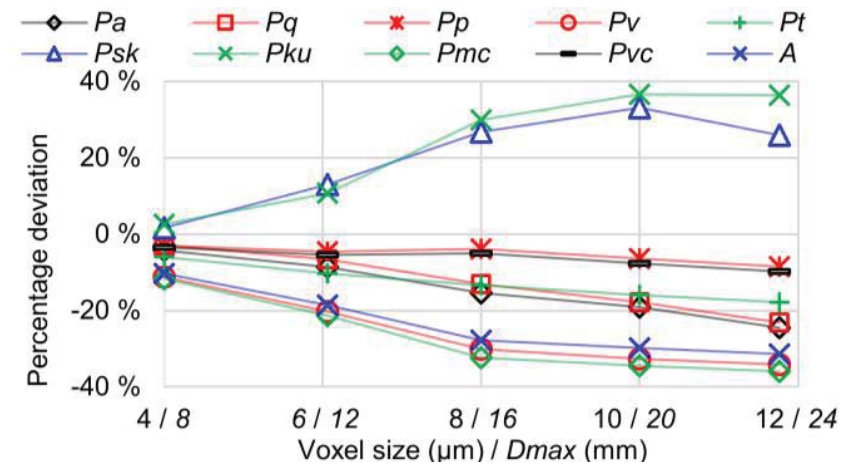

Figure 5. Deviations between generalised profile texture parameters measured by CT with different voxel sizes and on the corresponding reference profile. Maximum sample sizes Dmax are reported for a detector with a $2000 \times 2000$ grid of pixels with size of $0.2 \mathrm{~mm}$.

Table 2. Expanded uncertainties (95\% confidence) for P-parameters measured from CT data (voxel size $4 \mu \mathrm{m}$ ). All the values are expressed in micrometres. Ratios between uncertainties and measured mean values are reported in percentage.

\begin{tabular}{|c|c|c|c|c|c|}
\hline P-parameter & $P a$ & $P q$ & $P p$ & $P v$ & $P t$ \\
\hline$U$ & 2.4 & 2.4 & 5.4 & 10.0 & 12.5 \\
\hline$U \%$ & $22 \%$ & $17 \%$ & $9 \%$ & $34 \%$ & $14 \%$ \\
\hline
\end{tabular}

\subsection{Uncertainty determination}

The practical approach proposed in the guideline VDI/VDE 2630-2.1 [13] for determining the task-specific uncertainty of CT measurements, is here adapted to assess the uncertainty of CT surface profile measurements. The approach is based on repeated CT measurements and their comparison with reference data, which in this case are the reference profiles measured as explained in Section 4. In particular, the following formula is given in [13]:

$U=k \sqrt{u_{c a l}^{2}+u_{d r i f t}^{2}+u_{p}^{2}+u_{w}^{2}+u_{b}^{2}}$

where $k$ is the coverage factor (for a $95 \%$ confidence interval), $u_{c a l}$ is the standard uncertainty of the calibrated values, $u_{\text {drift }}$ is the uncertainty contribution of possible changes in the workpiece shape since the calibration referred to (which is negligible in this case, because the calibration and the measurements were performed in a short period), $u_{p}$ is measured as the standard deviation of the repeated CT measurements, $u_{w}$ is the standard uncertainty related to variations in materials and production, and $u_{b}$ is the uncertainty related to the correction of the systematic error $b$ (calculated as difference between mean measured value and calibrated reference value). As stated in [13], in cases where such correction is not possible, $\mathrm{b}$ should replace $u_{b}$ in formula (7). The influence of the cutting/polishing operations was taken into account by comparing the corresponding cross-sectional profiles measured by CT before and after such operations and included in the uncertainty. The influence of the background noise on the parameters' computation from the CT data is also considered and added to the uncertainty. Table 2 reports the final expanded uncertainties obtained with this approach for a selection of Pparameters, computed for the best resolution identified in Section $5.1(v s=4 \mu \mathrm{m})$. The relatively high value of uncertainty for $P v$ is mainly due to the limited capability of CT in detecting the maximum valley depths when the achieved structural resolution is not sufficiently high. The uncertainty worsens for increasing voxel sizes, for example up to $55 \%$ in the case of $P a$ computed for the worst resolution identified in Section $5.1(v s=12 \mu \mathrm{m})$.

\section{Conclusions}

The aim of this work was to improve the understanding of CTbased surface topography characterisation, including the use of new generalised surface texture parameters suited for AM surfaces, which are unconventionally characterised by the presence of re-entrant features. Experimental investigations were performed on Ti6Al4V L-PBF reference samples to determine the uncertainty of CT surface topography measurements when computing these newly defined parameters on CT data without removing re-entrant features. The reference samples were characterised by calibrated 2D cross-sectional profiles representing the actual morphology. The generalised parameters were compared with the conventional parameters on specific AM profiles and surfaces (respectively before and after removing the re-entrant features) and significant differences emerged with reference to all the investigated parameters, proving the importance of considering the actual surface morphology when evaluating the surface topography of metal AM parts. X-ray computed tomography was determined to be capable of acquiring profiles containing also re-entrant features, with deviations below $10 \%$ for the majority of the investigated profile texture parameters, and with uncertainties as reported in Table 2, for the investigated case and at the highest tested resolution (i.e. voxel size equal to $4 \mu \mathrm{m}$ ). Even if in the considered example the percentage of re-entrant features (Prf) was found to be $2.2 \%$ against the $6.2 \%$ of the reference profile, CT was determined to provide a clearly more complete description of the actual surface profile with respect to optical techniques (e.g. confocal microscopy). Future work is planned to improve the CT scanning parameters in order to further reduce the measurement errors and uncertainty.

\section{Acknowledgments}

The authors would like to thank professors Xiangqian Jiang and Paul J. Scott, University of Huddersfield, for valuable suggestions and inspiration, as well as Marco Sorgato and Elia Sbettega, University of Padua, for contributing to the experimental work. LP acknowledges the UKs Engineering and Physical Sciences Research Council (EPSRC) funding the grant Ref. EP/R024162/1.

\section{References}

[1] Schmidt $M$ et al (2017). Laser based additive manufacturing in industry and academia. CIRP Annals Man. Tech. 66/2:561-583.

[2] Carmignato $S$ et al (2017). Influence of surface roughness on computed tomography dimensional measurements. CIRP Annals Man. Tech. 66/1:499-502.

[3] Townsend A et al (2016). Surface texture metrology for metal additive manufacturing: a review. Precision Engineering 46 34-47

[4] ISO 25178-2:2012, GPS - Surface texture: Areal - Part 2: Terms, definitions and surface texture parameters.

[5] Thompson A et al (2017). Topography of selectively laser melted surfaces: A comparison of different measurement methods. CIRP Annals Man. Tech. 66/1

[6] Zanini F et al (2019). New approach for verifying the accuracy of X-ray computed tomography measurements of surface topographies in additively manufactured metal parts. Journal of Nondestructive Evaluation, 38/1, 12 .

[7] De Chiffre L et al (2014). Industrial applications of computed tomography. CIRP Annals Man. Tech., 63/2:655-677.

[8] Townsend A et al (2017). Factors affecting the accuracy of areal surface texture data extraction from X-ray CT. CIRP Annals Man. Tech. 66/1:547-550.

[9] Zanini F, Carmignato S (2017). Two-spheres method for evaluating the metrological structural resolution in dimensional computed tomography. Meas. Sci. and Tech., 28/11, 114002

[10] ISO 4288:1996, GPS - Surface Texture: Profile method - rules and procedures for the assessment of surface texture.

[11] Pagani L, Qi Q Jiang X, Scott P J (2017), Towards a new definition of areal surface texture parameters on freeform surface. Measurement, 109:281-291.

[12] Abbott E J, Firestone F A (1933). Specifying surface quality: a method based on accurate measurement and comparison. Mechanical Engineering, 55:569-572.

[13] VDI/VDE 2630 - 2.1:2015 - Computed tomography in dimensional

measurement - Determination of the uncertainty of measurement and the test process suitability of coordinate measurement systems with CT sensors. 